\title{
Inequalities involving Mittag-Leffler type $q$-Konhauser polynomial
}

\author{
Bharti Vishandas Nathwani
}

\begin{abstract}
In the present work, we propose generalized structure of the $q$ Konhauser polynomial suggested by a generalized $q$-Mittag-Leffler function. For this polynomial, we obtain its difference equation and several other properties involving inequalities are also derived which yield as the particular cases, $q$ analogues of the generating function relations and finite summation formulas.
\end{abstract}

Mathematics Subject Classification (2010): 33B15, 33E12, 33E99.

Keywords: $q$-Mittag-Leffler function, $q$-Konhauser polynomial, series inequalities, difference equation, generating function relation, series inequality relations.

\section{Introduction}

In 1903, Mittag-Leffler [20] proposed a function $E_{\alpha}(z)$ defined by

$$
E_{\alpha}(z)=\sum_{n=0}^{\infty} \frac{z^{n}}{\Gamma(\alpha n+1)},
$$

where $z$ is a complex variable and $\alpha \in \mathbb{C}, \Re(\alpha)>0$. Later on this function was referred to as Mittag-Leffler function. The Mittag-Leffler function is direct generalization of the exponential function to which it reduces for $\alpha=1$. This function has some interesting properties which later became essential for the description of many problems arising in applications. Nowadays the Mittag-Leffler function and its numerous generalizations have acquired a new life. The recent notable increased interest in the study of their relevant properties is due to the close connection to the Fractional Calculus and its application to the study of Differential and Integral Equations. Many modern models of fractional type have recently been proposed in Probability Theory, Mechanics, Mathematical Physics, Chemistry, Biology, Mathematical Economics, Engineering and Applied Sciences etc. There are many applications of Mittag-Leffler function and its generalizations in Astrophysics problems (see [17]). One application of MittagLeffler function is described below. 
In a reaction-diffusion process if $N(t)$ is the number density at a time $t$ and if the production rate is proportional to original number, then

$$
\frac{d}{d t} N(t)=\lambda N(t), \lambda>0
$$

where $\lambda$ is the rate of production. If the consumption or destruction rate is also proportional to the original number then

$$
\frac{d}{d t} N(t)=-\mu N(t), \quad \mu>0
$$

where $\mu$ is the destruction rate. Then the residual part is given by

$$
\frac{d}{d t} N(t)=-c N(t), \quad c=\mu-\lambda .
$$

If $c$ is free of $t$ then the solution is exponential model

$$
N(t)=N_{0} e^{-c t}, \quad N_{0}=N(t) \text { at } t=t_{0}
$$

where $t_{0}$ is the starting time. Instead of total derivative in (1.1) to (1.3) if the fractional derivative or fractional nature of reactions is considered, that is, an equation of the form

$$
N(t)-N_{0}=-c_{0}^{v} D_{t}^{-v} N(t)
$$

is considered where ${ }_{0} D_{t}^{-v}$ is the standard Riemann-Liouville fractional integral operator, then the solution

$$
N(t)=N_{0} \sum_{n=0}^{\infty} \frac{(-1)^{k}(c t)^{v k}}{\Gamma(v k+1)}=N_{0} E_{v}\left(-(c t)^{v}\right),
$$

involves $E_{v}($.$) which is nothing but the Mittag-Leffler function.$

The well known Mittag-Leffler function [20]

$$
E_{\alpha}(z)=\sum_{n=0}^{\infty} \frac{z^{n}}{\Gamma(\alpha n+1)}
$$

where $z$ is a complex variable and $\alpha \in \mathbb{C}, \Re(\alpha)>0$ was generalized by Wiman [37] in 1905 in the form:

$$
E_{\alpha, \beta}(z)=\sum_{n=0}^{\infty} \frac{z^{n}}{\Gamma(\alpha n+\beta)}, \quad \Re(\alpha, \beta)>0,
$$

which is known as Wiman's function or generalized Mittag-Leffler function.

Note 1.1. $E_{\alpha, 1}(z)=E_{\alpha}(z)$.

In 1971, Prabhakar [25] introduced its extension:

$$
E_{\alpha, \beta}^{\gamma}(z)=\sum_{n=0}^{\infty} \frac{(\gamma)_{n}}{\Gamma(\alpha n+\beta)} \frac{z^{n}}{n !},
$$

wherein $\Re(\alpha, \beta, \gamma)>0$.

Note 1.2. $E_{\alpha, \beta}^{1}(z)=E_{\alpha, \beta}(z)$. 
In 2007, Shukla and Prajapati [34] introduced the function:

$$
E_{\alpha, \beta}^{\gamma, q}(z)=\sum_{n=0}^{\infty} \frac{(\gamma)_{q n}}{\Gamma(\alpha n+\beta)} \frac{z^{n}}{n !},
$$

in which $\alpha, \beta, \gamma \in \mathbb{C} ; \Re(\alpha, \beta, \gamma)>0$ and $q \in(0,1) \cup \mathbb{N}$.

Note 1.3. $E_{\alpha, \beta}^{\gamma, 1}(z)=E_{\alpha, \beta}^{\gamma}(z)$.

As a continuation of these studies, Nathwani, Dave and Prajapati [27, 28, 29, 23, 22, 24] introduced the following function:

Definition 1.4. For $\alpha, \beta, \gamma, \lambda \in \mathbb{C}, \Re(\alpha, \beta, \gamma, \lambda)>0, \delta, \mu>0, r \in\{-1,0\} \cup \mathbb{N}, s \in$ $\mathbb{N} \cup\{0\}$

$$
E_{\alpha, \beta, \lambda, \mu}^{\gamma, \delta}(z ; s, r)=\sum_{n=0}^{\infty} \frac{\left[(\gamma)_{\delta n}\right]^{s}}{\Gamma(\alpha n+\beta)\left[(\lambda)_{\mu n}\right]^{r} n !} z^{n}
$$

Note 1.5. $E_{\alpha, \beta, \lambda, \mu}^{\gamma, q}(z ; 1,0)=E_{\alpha, \beta}^{\gamma, q}(z)$.

The objective of constructing this function is to

(i) include certain existing generalizations of Mittag-Leffler function,

(ii) also include the functions such as Bessel Maitland function, Dotsenko function, Bessel function, generalized Bessel Maitland function, Lommel function etc. especially by means of parameters $r, \gamma, \lambda$ (Table- 1 below)

(iii) obtain inverse inequality relations and some other inequalities by means of the integer ' $s$ '.

Since the time of Wiman (1905), many researchers have proposed and studied various generalizations of the Mittag-Leffler function [20] (see [38], [25], [34], [14], [15], [18], [21], [27], [29], [32], [33], [10]).

The $q$-analogue of the above generalized Mittag-Leffler function (1.11) is given by Nathwani and Dave [22, 24]:

$$
E_{\alpha, \beta, \lambda, \mu}^{\gamma, \delta}(z ; s, r \mid q)=\sum_{n=0}^{\infty} \frac{(-1)^{p n} q^{p n(n-1) / 2}\left[\Gamma_{q}(\gamma+\delta n)\right]^{s}}{\Gamma_{q}(\beta+\alpha n)\left[\Gamma_{q}(\lambda+\mu n)\right]^{r}(q ; q)_{n}} z^{n}
$$

where $\alpha, \beta, \gamma, \lambda \in \mathbf{C}$ with $\Re(\alpha, \beta, \gamma, \lambda)>0, \delta, \mu>0, r \in\{-1,0\} \cup \mathbf{N}, s \in \mathbf{N} \cup\{0\}$, $p=\alpha^{2}+r \mu^{2}-s \delta^{2}+1$ with $\Re(p)>0$.

The aim of the present work is to extend the well known $q$-Konhauser Polynomial [6]

$$
Z_{m}^{\beta}(x ; k \mid q)=\frac{\left[q^{\beta+1}\right]_{k m}}{\left(q^{k} ; q^{k}\right)_{m}} \sum_{n=0}^{m} \frac{q^{k n(k n-1) / 2+k n(m+\beta+1)}\left(q^{-m k} ; q^{k}\right)_{n} x^{k n}}{\left[q^{\beta+1}\right]_{k n}\left(q^{k} ; q^{k}\right)_{n}}
$$

and hence the generalized $q$-Laguerre polynomial [30]:

$$
L_{m}^{(\beta)}(x \mid q)=\frac{\left[q^{\beta+1}\right]_{m}}{(q ; q)_{m}} \sum_{n=0}^{m} \frac{q^{n(n+1) / 2+n(m+\beta)}\left(q^{-m} ; q\right)_{n} x^{n}}{\left[q^{\beta+1}\right]_{n}(q ; q)_{n}},
$$

where $\Re(\beta)>-1$ suggested by the generalized $q$-Mittag-Leffler function (1.12).

The following definitions and formulas will be used in this work. 
For $a \in \mathbf{C}$, and $0<|q|<1$, the $q$-shifted factorial is defined by [13, Eq.(1.2.15), p.3 and Eq.(1.2.30), p.6]

$$
(a ; q)_{n}= \begin{cases}1 & \text { if } n=0 \\ (1-a)(1-a q) \cdots\left(1-a q^{n-1}\right) & \text { if } n \in \mathbf{N} \\ \frac{(q ; q)_{\infty}}{\left(a q^{n} ; q\right)_{\infty}} & \text { if } n \in \mathbf{C}\end{cases}
$$

where

$$
(a ; q)_{\infty}=\prod_{k=0}^{\infty}\left(1-a q^{k}\right), \quad|q|<1 .
$$

A well-known extension of the $q$-shifted factorial is given by [12]

$$
[t-\mid a]_{n}=(t-a)(t-a q)\left(t-a q^{2}\right) \cdots\left(t-a q^{n-1}\right) .
$$

A finite series-product identity [12]

$$
\sum_{k=0}^{n} q^{k(k-1) / 2}\left[\begin{array}{l}
n \\
k
\end{array}\right] x^{k}=\prod_{k=1}^{n}\left(1+x q^{k-1}\right) .
$$

The $q$-derivative of a function $f(x)$ is defined by [13, Ex.1.12, p.22]

$$
D_{q} f(x)=\frac{f(x)-f(x q)}{x(1-q)}
$$

Definition 1.6. A $q$-Gamma function is defined as ([16], [35]):

$$
\Gamma_{q}(\alpha)=\frac{(q ; q)_{\infty}(1-q)^{1-\alpha}}{\left(q^{\alpha} ; q\right)_{\infty}},
$$

where $\alpha \neq 0,-1,-2, \ldots$, and $0<q<1$.

The $q$-analogue of Stirling's asymptotic formula [19, Eq.(2.25), p.482] for the $q$ Gamma function is

$$
\Gamma_{q}(x) \sim(1+q)^{\frac{1}{2}} \Gamma_{q^{2}}\left(\frac{1}{2}\right)(1-q)^{\frac{1}{2}-x} e^{\mu_{q}(x)},
$$

where $\mu_{q}(x)=\frac{\theta q^{x}}{1-q-q^{x}}, 0<\theta<1$.

A $q$-Beta function $\mathbf{B}_{q}(x, y)$ is expressible in different ways [13].

$$
\begin{gathered}
\mathbf{B}_{q}(x, y)=\int_{0}^{1} t^{x-1}(t q)_{y-1} \mathrm{~d}_{\mathrm{q}} \mathrm{t}, \\
\mathbf{B}_{q}(x, y)=\frac{(1-q)(q)_{\infty}\left(q^{x+y}\right)_{\infty}}{\left(q^{x}\right)_{\infty}\left(q^{y}\right)_{\infty}},
\end{gathered}
$$

and

$$
\mathbf{B}_{q}(x, y)=\int_{0}^{1} t^{x-1} \frac{(t q ; q)_{\infty}}{\left(t q^{y} ; q\right)_{\infty}} \mathrm{d}_{\mathrm{q}} \mathrm{t}
$$


in which $y \neq 0,-1,-2, \ldots, \Re(x)>0$.

The $q$-Euler (Beta) transform is [13]:

$$
\mathbf{B}\{f(z): a, b \mid q\}=\int_{0}^{1} u^{\beta-1} \frac{(u q ; q)_{\infty}}{\left(u q^{\eta} ; q\right)_{\infty}} f(z) \mathrm{d}_{\mathrm{q}} \mathrm{u} .
$$

The $q$-Laplace transform.

Hahn [16] defined the q-analogues of the well known classical Laplace transform:

$$
F(S)=\phi(S)=\int_{0}^{\infty} e^{-S t} f(t) \mathrm{dt},
$$

by means of the following two integral equations.

$$
\mathcal{L}_{q}\{f(t)\}=\frac{1}{(1-q)} \int_{0}^{S_{-1}^{-1}} E_{q}(q S t) f(t) \mathrm{d}_{\mathrm{q}} \mathrm{t},
$$

and

$$
\mathcal{L}_{q}\{f(t)\}=\frac{1}{(1-q)} \int_{0}^{\infty} e_{q}(-S t) f(t) \mathrm{d}_{\mathrm{q}} \mathrm{t}
$$

where $\Re(S)>0$.

The $q$-analogue of Riemann-Liouville fractional integral operator ([4], [39], [31]) is given by

$$
{ }_{q} I_{a+}^{\mu} f(x)=\frac{1}{\Gamma_{q}(\mu)} \int_{a}^{x}(x-\mid y q)_{\mu-1} f(y) \mathrm{d}_{q} y,
$$

where $\mu$ is an arbitrary order of integration with $\Re(\mu)>0$.

In particular, for $f(x)=x^{\nu-1}$, the equation (1.26) reduces to

$$
{ }_{q} I_{0+}^{\mu} f(x)\left[x^{\nu-1}\right]=\frac{\Gamma_{q}(\nu)}{\Gamma_{q}(\nu+\mu)} x^{\nu+\mu-1} .
$$

The fractional $q$-differential operator of arbitrary order $\alpha$, is defined as ([5], [31]):

$$
\left({ }_{q} D_{0+}^{\alpha} f\right)(x)=\frac{1}{\Gamma_{q}(-\alpha)} \int_{0}^{x}(x-\mid y q)_{-\alpha-1} f(y) \mathrm{d}_{q} y,
$$

in which $\Re(\alpha)<0,0<|q|<1$.

It is to be noted that $\left({ }_{q} D_{0+}^{\alpha} f\right)(x)=D_{x, q}^{\alpha} f(x)$. In this context, we have

$$
\left({ }_{q} D_{a+}^{\alpha} f\right)(x)=\left(\frac{d_{q}}{d_{q} x}\right)^{n}\left({ }_{q} I_{a+}^{n-\alpha} f\right)(x) .
$$

If $f(x)=x^{\mu-1}$, then $(1.28)$ reduces to

$$
{ }_{q} D_{0+}^{\alpha}\left[x^{\mu-1}\right]=\frac{\Gamma_{q}(\mu)}{\Gamma_{q}(\mu-\alpha)} x^{\mu-\alpha-1} .
$$


The generalized Konhauser polynomial due to Prajapati, Ajudia and Agarwal is given by $[26]$ :

$$
L_{m^{*}}^{(k, \sigma)}\left(x^{k}\right)=Z_{m^{*}}^{\sigma}(x ; k)=\frac{\Gamma(k m+\sigma+1)}{\Gamma(m+1)} \sum_{n=0}^{m^{*}} \frac{(-m)_{\delta n}}{\Gamma(k n+\sigma+1)} \frac{x^{k n}}{n !} .
$$

We propose here generalization of $q$-Konhauser polynomial.

Definition 1.7. For $\alpha, \beta, \lambda>0, m, \delta, \mu, k, s \in \mathbf{N}, r \in \mathbf{N} \cup\{0\}, m^{*}=\left[\frac{m}{\delta}\right]$, the integral part of $\frac{m}{\delta}$, define

$$
\begin{aligned}
B_{m^{*}}^{(\alpha, \beta, \lambda, \mu)}\left(x^{k} ; s, r \mid q\right)= & \frac{\left(q^{\beta+1} ; q\right)_{\alpha m}}{\left[\left(q^{k} ; q^{k}\right)_{m}\right]^{3}} \sum_{n=0}^{m^{*}} \frac{q^{s k \delta n(m+(\delta n k-1) / 2)} q^{\delta n(\alpha(\beta+1)+r \mu \lambda)}}{\left(q^{\beta+1} ; q\right)_{\alpha n}\left[\left(q^{\lambda} ; q\right)_{\mu n}\right]^{r}} \\
& \times \frac{\left[\left(q^{-m k} ; q^{k}\right)_{\delta n}\right]^{s} x^{k n}}{\left(q^{k} ; q^{k}\right)_{n}}
\end{aligned}
$$

The polynomial in (1.32) will be referred to as $q$-GKP.

\section{Generalized $q$-Konhauser polynomial}

If $\alpha=k \in \mathbf{N}, s=1, r=0$ then (1.32) reduces to $q$-analogue of another generalization of the Konhauser polynomial (1.31) in the form considered by [26]:

$$
\begin{aligned}
B_{m^{*}}^{(k, \beta, \lambda, \mu)}\left(x^{k} ; 1,0 \mid q\right)= & \frac{\left(q^{\beta+1} ; q\right)_{k m}}{\left(q^{k} ; q^{k}\right)_{m}} \sum_{n=0}^{m^{*}} \frac{q^{k \delta n(k \delta n-1) / 2+\delta n m} q^{\delta n k(\beta+1)}}{\left(q^{\beta+1} ; q\right)_{\alpha n}} \\
& \times \frac{\left(q^{-m k} ; q^{k}\right)_{\delta n} x^{k n}}{\left(q^{k} ; q^{k}\right)_{n}} \\
= & Z_{m^{*}}^{\beta}(x ; k \mid q) .
\end{aligned}
$$

A $q$-analogue of the classical Konhauser polynomial (1.13) is obtained from (2.1) by taking $\delta=1$, that is

$$
\begin{aligned}
B_{m}^{(k, \beta, \lambda, \mu)}\left(x^{k} ; 1,0 \mid q\right) & =\frac{\left(q^{\beta+1} ; q\right)_{k m}}{\left(q^{k} ; q^{k}\right)_{m}} \sum_{n=0}^{m} \frac{q^{k n(k n-1) / 2+k n(m+\beta+1)}\left(q^{-m k} ; q^{k}\right)_{n} x^{k n}}{\left(q^{\beta+1} ; q\right)_{k n}\left(q^{k} ; q^{k}\right)_{n}} \\
& =Z_{m}^{\beta}(x ; k \mid q) .
\end{aligned}
$$

Further, with $k=1$,

$$
\begin{aligned}
B_{m}^{(1, \beta, \lambda, \mu)}(x ; 1,0 \mid q) & =\frac{\left(q^{\beta+1} ; q\right)_{m}}{(q ; q)_{m}} \sum_{n=0}^{m} \frac{q^{n(n+1) / 2+n(m+\beta)}\left(q^{-m} ; q\right)_{n} x^{n}}{\left(q^{\beta+1} ; q\right)_{n}(q ; q)_{n}} \\
& =L_{m}^{(\beta)}(x \mid q)
\end{aligned}
$$

is a $q$-analogue of the generalized Laguerre polynomial. 
Theorem 2.1. Let

$$
\begin{aligned}
B_{m^{*}}^{(\alpha, \beta, \lambda, \mu)}\left(x^{k} ; s, r \mid q\right)= & \frac{\left(q^{\beta+1} ; q\right)_{\alpha m}}{\left[\left(q^{k} ; q^{k}\right)_{m}\right]^{s}} \sum_{n=0}^{m^{*}} \frac{q^{s(k \delta n(k \delta n-1) / 2+k \delta n m)} q^{\delta n(\alpha(\beta+1)+r \mu \lambda)}}{\left(q^{\beta+1} ; q\right)_{\alpha n}\left[\left(q^{\lambda} ; q\right)_{\mu n}\right]^{r}} \\
& \times \frac{\left[\left(q^{-m k} ; q^{k}\right)_{\delta n}\right]^{s} x^{k n}}{\left(q^{k} ; q^{k}\right)_{n}} .
\end{aligned}
$$

Then as limit $m \rightarrow \infty, B_{m^{*}}^{(\alpha, \beta, \lambda, \mu)}\left(x^{k} ; s, r \mid q\right)$ approaches to the entire function

$$
\begin{aligned}
B_{\infty}^{(\alpha, \beta, \lambda, \mu)}\left(x^{k} ; s, r \mid q\right)= & \frac{\left(q^{\beta+1} ; q\right)_{\infty}}{\left[\left(q^{k} ; q^{k}\right)_{\infty}\right]^{s}} \sum_{n=0}^{\infty} \frac{(-1)^{s \delta n} q^{s(k \delta n(k \delta n-1) / 2+k \delta n(\delta n-1) / 2)}}{\left(q^{\beta+1} ; q\right)_{\alpha n}\left[\left(q^{\lambda} ; q\right)_{\mu n}\right]^{r}} \\
& \times \frac{q^{\delta n(\alpha(\beta+1)+r \mu \lambda)} x^{k n}}{\left(q^{k} ; q^{k}\right)_{n}}
\end{aligned}
$$

in any bounded domain.

Proof. It will be shown first that the series in (2.5) has an infinite radius of convergence.

Taking

$$
\begin{aligned}
v_{n}= & \frac{(-1)^{s \delta n} q^{s(k \delta n(k \delta n-1) / 2+k \delta n(\delta n-1) / 2)} \frac{q^{\delta n(\alpha(\beta+1)+r \mu \lambda)}}{\left(q^{\beta+1} ; q\right)_{\alpha n}\left[\left(q^{\lambda} ; q\right)_{\mu n}\right]^{r}}}{\left(q^{k} ; q^{k}\right)_{n}} \\
= & \frac{(-1)^{s \delta n} q^{s(k \delta n(k \delta n-1) / 2+k \delta n(\delta n-1) / 2)} q^{\delta n(\alpha(\beta+1)+r \mu \lambda)}}{\left(q^{\beta+1} ; q\right)_{\infty}\left[\left(q^{\lambda} ; q\right)_{\infty}\right]^{r}} \\
& \times \frac{\left(q^{\alpha n+\beta+1} ; q\right)_{\infty}\left[\left(q^{\mu n+\lambda} ; q\right)_{\infty}\right]^{r}}{\left(q^{k} ; q^{k}\right)_{n}} .
\end{aligned}
$$

Then using D'Albert's Ratio test, the radius of convergence $R$ is given by

$$
\begin{aligned}
R= & \lim _{n \rightarrow \infty}\left|\frac{v_{n}}{v_{n+1}}\right| \\
= & \lim _{n \rightarrow \infty} \mid \frac{(-1)^{s \delta n} q^{s(k \delta n(k \delta n-1) / 2+k \delta n(\delta n-1) / 2)} q^{\delta n(\alpha(\beta+1)+r \mu \lambda)}}{\left(q^{\beta+1} ; q\right)_{\infty}\left[\left(q^{\lambda} ; q\right)_{\infty}\right]^{r}\left(q^{k} ; q^{k}\right)_{n}} \\
& \times \frac{\left(q^{\beta+1} ; q\right)_{\infty}\left[\left(q^{\lambda} ; q\right)_{\infty}\right]^{r}\left(q^{\alpha n+\beta+1} ; q\right)_{\infty}\left[\left(q^{\mu n+\lambda} ; q\right)_{\infty}\right]^{r}}{(-1)^{s \delta(n+1)} q^{s k \delta(n+1)(\delta(n+1)-1) / 2+(k \delta(n+1)-1) / 2}} \\
& \times \frac{\left(q^{k} ; q^{k}\right)_{n+1}}{q^{\delta(n+1)(\alpha(\beta+1)+r \mu \lambda)}\left(q^{\alpha(n+1)+\beta+1} ; q\right)_{\infty}\left[\left(q^{\mu(n+1)+\lambda} ; q\right)_{\infty}\right]^{r}} \mid \\
= & \lim _{n \rightarrow \infty} \mid \frac{q^{s k \delta-s k^{2} \delta^{2}}\left(1-q^{(n+1) k}\right)}{q^{n s \delta^{2}(k(k+1))} q^{\delta(\alpha(\beta+1)+r \mu \lambda)}} \\
& \times \frac{\left(1-q^{\alpha n+\beta+1}\right)\left(1-q^{\alpha n+\beta+2}\right) \ldots\left(1-q^{\alpha n+\beta+\alpha}\right)}{\left[\left(1-q^{\mu n+\lambda+1}\right)\left(1-q^{\mu n+\lambda+2}\right) \ldots\left(1-q^{\mu n+\lambda+\mu}\right)\right]^{-r}} \mid \\
= & \infty .
\end{aligned}
$$


Here it suffices to show that for $m$ sufficiently large,

$$
\sum_{n=0}^{m^{*}} \frac{q^{s(k \delta n(k \delta n-1) / 2+k \delta n m)} q^{\delta n(\alpha(\beta+1)+r \mu \lambda)}}{\left(q^{\beta+1} ; q\right)_{\alpha n}\left[\left(q^{\lambda} ; q\right)_{\mu n}\right]^{r}} \frac{\left[\left(q^{-m k} ; q^{k}\right)_{\delta n}\right]^{s} x^{k n}}{\left(q^{k} ; q^{k}\right)_{n}}
$$

tends to

$$
\sum_{n=0}^{\infty} \frac{(-1)^{s \delta n} q^{s(k \delta n(k \delta n-1) / 2+k \delta n(\delta n-1) / 2)}}{\left(q^{\beta+1} ; q\right)_{\alpha n}\left[\left(q^{\lambda} ; q\right)_{\mu n}\right]^{r}} \frac{q^{\delta n(\alpha(\beta+1)+r \mu \lambda)} x^{k n}}{\left(q^{k} ; q^{k}\right)_{n}}
$$

In fact,

$$
\begin{aligned}
& \mid \sum_{n=0}^{\infty} \frac{(-1)^{s \delta n} q^{s(k \delta n(k \delta n-1) / 2+k \delta n(\delta n-1) / 2)}}{\left(q^{\beta+1} ; q\right)_{\alpha n}\left[\left(q^{\lambda} ; q\right)_{\mu n}\right]^{r}} \frac{q^{\delta n(\alpha(\beta+1)+r \mu \lambda)} x^{k n}}{\left(q^{k} ; q^{k}\right)_{n}} \\
&-\sum_{n=0}^{m^{*}} \frac{q^{s(k \delta n(k \delta n-1) / 2+k \delta n m)} q^{\delta n(\alpha(\beta+1)+r \mu \lambda)}}{\left(q^{\beta+1} ; q\right)_{\alpha n}\left[\left(q^{\lambda} ; q\right)_{\mu n}\right]^{r}} \frac{\left[\left(q^{-m k} ; q^{k}\right)_{\delta n}\right]^{s} x^{k n}}{\left(q^{k} ; q^{k}\right)_{n}} \mid \\
&= \mid \sum_{n=0}^{m^{*}}\left\{q^{s k \delta n(\delta n-1) / 2}-\left[\left(q^{-m k} ; q^{k}\right)_{\delta n}\right]^{s} q^{s k \delta n}(-1)^{s \delta n}\right\} \\
&= \times \frac{q^{s k \delta n(k \delta n-1) / 2} q^{\delta n(\alpha(\beta+1)+r \mu \lambda)}(-1)^{s \delta n} x^{k n}}{\left(q^{\beta+1} ; q\right)_{\alpha n}\left[\left(q^{\lambda} ; q\right)_{\mu n}\right]^{r}\left(q^{k} ; q^{k}\right)_{n}} \mid \\
&\left.\left.\times\left(1-q^{-m k+(\delta n-1) k}\right)\right]^{s} q^{s k \delta n m}(-1)^{s \delta n}\right\} \\
&= \times \frac{q^{s k \delta n(k \delta n-1) / 2} q^{\delta n(\alpha(\beta+1)+r \mu \lambda)}(-1)^{s \delta n} x^{k n}}{\left(q^{\beta+1} ; q\right)_{\alpha n}\left[\left(q^{\lambda} ; q\right)_{\mu n}\right]^{r}\left(q^{k} ; q^{k}\right)_{n}} \mid \\
& \times \frac{q^{s k \delta n(\delta n-1) / 2}-\left[\left(1-q^{-m k}\right)\left(1-q^{-m k+k}\right)\left(1-q^{-m k+2 k}\right) \ldots\right.}{\left(q^{\beta+1} ; q\right)_{\alpha n}\left[\left(q^{\lambda} ; q\right)_{\mu n}\right]^{r}\left(q^{k} ; q^{k}\right)_{n}} \mid \\
&\left.\left.\times\left(q^{-m k+(\delta n-1) k}-1\right)\right]^{s} q^{s k \delta n m}\right\} \\
& m^{*}\left\{q^{s k \delta n(\delta n-1) / 2}-\left[\left(q^{-m k}-1\right)\left(q^{-m k+k}-1\right)\left(q^{-m k+2 k}-1\right) \ldots\right.\right.
\end{aligned}
$$




$$
\begin{aligned}
= & \mid \sum_{n=0}^{m^{*}}\left\{q^{s k \delta n(\delta n-1) / 2}-\left[\left(1-q^{m k}\right)\left(1-q^{m k-k}\right)\left(1-q^{m k-2 k}\right) \ldots\right.\right. \\
& \left.\left.\times\left(1-q^{m k-(\delta n-1) k}\right)\right]^{s} q^{s k \delta n m} q^{s k \delta n(\delta n-1) / 2-s k \delta n m}\right\} \\
& \times \frac{q^{s k \delta n(k \delta n-1) / 2} q^{\delta n(\alpha(\beta+1)+r \mu \lambda)}(-1)^{s \delta n} x^{k n}}{\left(q^{\beta+1} ; q\right)_{\alpha n}\left[\left(q^{\lambda} ; q\right)_{\mu n}\right]^{r}\left(q^{k} ; q^{k}\right)_{n}} \mid \\
\leq & \sum_{n=0}^{m^{*}} \mid q^{s k \delta n(\delta n-1) / 2}-\left[\left(1-q^{m k}\right)\left(1-q^{m k-k}\right)\left(1-q^{m k-2 k}\right) \ldots\right. \\
& \left.\times\left(1-q^{m k-(\delta n-1) k}\right)\right]^{s} q^{s k \delta n(\delta n-1) / 2} \mid \\
& \times \frac{q^{s k \delta n(k \delta n-1) / 2} q^{\delta n(\alpha(\beta+1)+r \mu \lambda)}|x|^{k n}}{\left(q^{\beta+1} ; q\right)_{\alpha n}\left[\left(q^{\lambda} ; q\right)_{\mu n}\right]^{r}\left(q^{k} ; q^{k}\right)_{n}} .
\end{aligned}
$$

The absolute difference may be simplified with the aid of the inequality

$$
\prod_{j=1}^{k}\left(1-x_{j}\right) \geq 1-\sum_{j=1}^{k} x_{j}, \quad 0 \leq x_{j} \leq 1, j=1,2, \ldots, k
$$

to get

$$
\begin{aligned}
& \mid q^{s k \delta n(\delta n-1) / 2}-\left[\left(1-q^{m k}\right)\left(1-q^{m k-k}\right)\left(1-q^{m k-2 k}\right) \ldots\right. \\
& \left.\times\left(1-q^{m k-(\delta n-1) k}\right)\right]^{s} q^{s k \delta n(\delta n-1) / 2} \mid \\
= & q^{s k \delta n(\delta n-1) / 2} \\
& \times\left|1-\left[\left(1-q^{m k}\right)\left(1-q^{m k-k}\right)\left(1-q^{m k-2 k}\right) \ldots\left(1-q^{m k-(\delta n-1) k}\right)\right]^{s}\right| \\
= & q^{s k \delta n(\delta n-1) / 2}\left|1-\left[\prod_{j=1}^{\delta n}\left(1-q^{m k-j k+k}\right)\right]^{s}\right| \\
\leq & q^{s k \delta n(\delta n-1) / 2}\left|1-\left(1-\sum_{j=1}^{\delta n} q^{m k-j k+k}\right)^{s}\right| \\
\leq & q^{s k \delta n(\delta n-1) / 2}\left|\sum_{j=1}^{\delta n} q^{m k-j k+k}\right|^{s} \mid
\end{aligned}
$$




$$
\begin{aligned}
& =q^{s k \delta n(\delta n-1) / 2}\left(\sum_{j=1}^{\delta n} q^{m k-j k+k}\right)^{s} \\
& =q^{s k \delta n(\delta n-1) / 2+s k m}\left(\sum_{j=0}^{\delta n-1} q^{-j k}\right)^{s} \\
& =q^{s k \delta n(\delta n-1) / 2+s k m} \frac{\left(1-q^{-\delta n k}\right)^{s}}{\left(1-q^{-k}\right)^{s}} \\
& =q^{s k \delta n(\delta n-1) / 2-s k \delta n+s m k+s k} \frac{\left(1-q^{\delta n k}\right)^{s}}{\left(1-q^{k}\right)^{s}} \\
& \leq \frac{q^{s k \delta n(\delta n-1) / 2-s k \delta n+s m k+s k}}{\left(1-q^{k}\right)^{s}}
\end{aligned}
$$

because $\delta n \leq m$. Therefore,

$$
\begin{aligned}
& \mid q^{s k \delta n(\delta n-1) / 2}-\left[\left(1-q^{m k}\right)\left(1-q^{m k-k}\right)\left(1-q^{m k-2 k}\right) \ldots\right. \\
& \left.\times\left(1-q^{m k-(\delta n-1) k}\right)\right]^{s} q^{s k \delta n(\delta n-1) / 2} \mid \\
\leq & \frac{q^{s k \delta n(\delta n-1) / 2-s n \delta k+s m k+s k}}{\left(1-q^{k}\right)^{s}} .
\end{aligned}
$$

This last inequality is valid for all non negative values of $\delta n$. Substituting this into (2.8), one gets

$$
\begin{aligned}
& \mid \sum_{n=0}^{\infty} \frac{(-1)^{s \delta n} q^{s(k \delta n(k \delta n-1) / 2+k \delta n(\delta n-1) / 2)}}{\left(q^{\beta+1} ; q\right)_{\alpha n}\left[\left(q^{\lambda} ; q\right)_{\mu n}\right]^{r}} \frac{q^{\delta n(\alpha(\beta+1)+r \mu \lambda)} x^{k n}}{\left(q^{k} ; q^{k}\right)_{n}} \\
& -\sum_{n=0}^{m^{*}} \frac{q^{s(k \delta n(k \delta n-1) / 2+k \delta n m)} q^{\delta n(\alpha(\beta+1)+r \mu \lambda)}}{\left(q^{\beta+1} ; q\right)_{\alpha n}\left[\left(q^{\lambda} ; q\right)_{\mu n}\right]^{r}} \frac{\left[\left(q^{-m k} ; q^{k}\right)_{\delta n}\right]^{s} x^{k n}}{\left(q^{k} ; q^{k}\right)_{n}} \mid \\
\leq & \frac{q^{s m k+s k}}{\left(1-q^{k}\right)^{s}} \sum_{n=0}^{\infty} \frac{q^{s k \delta n(\delta n-1) / 2-s n \delta k} q^{s k \delta n(k \delta n-1) / 2} q^{\delta n(\alpha(\beta+1)+r \mu \lambda)}|x|^{k n}}{\left(q^{\beta+1} ; q\right)_{\alpha n}\left[\left(q^{\lambda} ; q\right)_{\mu n}\right]^{r}\left(q^{k} ; q^{k}\right)_{n}}
\end{aligned}
$$

Thus the last series (2.10) has an infinite radius of convergence and is therefore bounded in every bounded domain. It follows that the left hand side in $(2.8) \rightarrow 0$ as $n \rightarrow \infty$ uniformly in any bounded domain. Hence the series (2.6) converges to (2.7) uniformly on any bounded domain.

\section{Difference equations}

The operators considered in this section are listed below.

$$
\Lambda_{q} f(x)=f(x)-f\left(x q^{-1}\right), \quad \Theta f(x)=f(x)-f(x q),
$$




$$
\begin{aligned}
\mathcal{D}_{q} f(x)= & (1-q) D_{q} f(x):=(1-q) \frac{f(x)-f(x q)}{x-x q}=\frac{f(x)-f(x q)}{x}, \\
& \frac{\left\{\prod_{u=0}^{a-1} \prod_{v=0}^{a-1}\left[\Theta+c^{-u} q^{1-(b+v) / a}-1\right]^{m}\right\}}{\left\{\prod_{u=0}^{a-1} \prod_{v=0}^{a-1}\left[c^{-u} q^{1-(b+v) / a}\right]^{m}\right\}}=\Phi_{u, v}^{(a, b, c ; m)}
\end{aligned}
$$

and

$$
\frac{\left\{\prod_{u=0}^{a-1} \prod_{v=0}^{a-1}\left[\Theta+c^{-u} q^{(b+v) / a}-1\right]^{m}\right\}}{\left\{\prod_{u=0}^{a-1} \prod_{v=0}^{a-1}\left[c^{-u} q^{-(b+v) / a}\right]^{m}\right\}}=\Psi_{u, v}^{(a, b, c ; m)} .
$$

In the notations of these operators, the difference equation satisfied by the polynomial $B_{m^{*}}^{(\alpha, \beta, \lambda, \mu)}\left(x^{k} ; s, r \mid q\right)$ is derived in the following theorem.

Theorem 3.1. Let $\alpha, \beta, \lambda, m, \delta, \mu, k, s \in \mathbf{N}, r \in \mathbf{N} \cup\{0\}, m^{*}=\left[\frac{m}{\delta}\right]$ then $B_{m^{*}}^{(\alpha, \beta, \lambda, \mu)}\left(x^{k} ; s, r \mid q\right)$ satisfies the equation

$$
\begin{aligned}
& {\left[\Phi_{\ell, \kappa}^{(\mu, \lambda, \eta ; r)} \Phi_{h, g}^{(\alpha, \beta+1, \sigma ; 1)} \Theta\right] B_{m^{*}}^{(\alpha, \beta, \lambda, \mu)}\left(x^{k} ; s, r \mid q\right)} \\
& -x^{k} q^{s(k \delta(k \delta-1) / 2)+s k \delta m} \Psi_{j, i}^{(\delta k,-m k, \chi ; s)} B_{m^{*}}^{(\alpha, \beta, \lambda, \mu)}\left(x^{k} q^{s(k \delta)^{2}} ; s, r \mid q\right)=0,
\end{aligned}
$$

where $\chi i s(\delta k)^{\text {th }}$ root of unity, $\eta$ is $\mu^{\text {th }}$ root of unity, $\sigma$ is $\alpha^{\text {th }}$ root of unity.

Proof. The coefficient of $x^{n k}$ in (1.32) will be first expressed in $q$-factorial notation with the aid of the formulas [13, Appendix I]:

$$
\begin{gathered}
(a ; q)_{k n}=\left(a, a q, \ldots, a q^{k-1} ; q^{k}\right)_{n} \\
\left(a^{k} ; q^{k}\right)_{n}=\left(a, a \omega_{k}, \ldots, a \omega_{k}^{k-1} ; q^{k}\right)_{n} ; \omega_{k}=e^{(2 \pi i) / k} \\
\left(A ; q^{n}\right)_{\nu k}=\left(A^{1 / n} ; q\right)_{\nu k}\left(A^{1 / n} \omega ; q\right)_{\nu k} \ldots\left(A^{1 / n} \omega^{n-1} ; q\right)_{\nu k}, \omega^{n}=1,
\end{gathered}
$$

and

$$
\left(q^{\gamma} ; q^{\delta}\right)_{n}=\left(q^{\gamma / \delta} ; q\right)_{n}\left(\varpi q^{\gamma / \delta} ; q\right)_{n} \ldots\left(\varpi^{\delta-1} q^{\gamma / \delta} ; q\right)_{n}=\prod_{i=0}^{\delta-1}\left(\varpi^{i} q^{\gamma / \delta} ; q\right)_{n}, \varpi^{\delta}=1 .
$$

Now if

$$
\begin{aligned}
& \sum_{n=0}^{[n / \delta]}\left\{\prod_{j=0}^{\delta k-1} \prod_{i=0}^{\delta k-1}\left[\left(\chi^{j} q^{(-m k+i) /(\delta k)} ; q\right)_{n}\right]^{s}\right\}\left\{\prod_{\ell=0}^{\mu-1} \prod_{\kappa=0}^{\mu-1}\left[\left(\eta^{\ell} q^{(\lambda+\kappa) / \mu} ; q\right)_{n}\right]^{r}\right\}^{-1} \\
& \times \frac{q^{s(k \delta n(k \delta n-1) / 2+k \delta n m)} q^{\delta n(\alpha(\beta+1)+r \mu \lambda)}}{\left\{\prod_{h=0}^{\alpha-1} \prod_{g=0}^{\alpha-1}\left(\sigma^{h} q^{(\beta+g) / \alpha} ; q\right)_{n}\right\}} \frac{x^{n k}}{\left(q^{k} ; q^{k}\right)_{n}}=\mathcal{Y}
\end{aligned}
$$




$$
\begin{gathered}
\prod_{j=0}^{\delta k-1} \prod_{i=0}^{\delta k-1}\left[\left(\chi^{j} q^{(-m k+i) /(\delta k)} ; q\right)_{n}\right]^{s}=\mathcal{H}_{n}, \prod_{\ell=0}^{\mu-1} \prod_{k=0}^{\mu-1}\left[\left(\eta^{\ell} q^{(\lambda+k) / \mu} ; q\right)_{n}\right]^{r}=\mathcal{B}_{n}, \\
\prod_{h=0}^{\alpha-1} \prod_{g=0}^{\alpha-1}\left(\sigma^{h} q^{(\beta+g) / \alpha} ; q\right)_{n}=\mathcal{C}_{n}, q^{s(k \delta n(k \delta n-1) / 2+k \delta n m)} q^{\delta n(\alpha(\beta+1)+r \mu \lambda)}=\mathcal{G}_{n},
\end{gathered}
$$

then (3.2) will assume the elegant form:

$$
\mathcal{Y}=\sum_{n=0}^{[n / \delta]} \frac{\mathcal{H}_{n} \mathcal{G}_{n}}{\mathcal{B}_{n} \mathcal{C}_{n}\left(q^{k} ; q^{k}\right)_{n}} x^{n k}
$$

Now

$$
\Theta \mathcal{Y}=\sum_{n=0}^{[n / \delta]} \frac{\mathcal{H}_{n} \mathcal{G}_{n}}{\mathcal{B}_{n} \mathcal{C}_{n}\left(q^{k} ; q^{k}\right)_{n}} \Theta x^{n k}=\sum_{n=1}^{[n / \delta]} \frac{\mathcal{H}_{n} \mathcal{G}_{n}}{\mathcal{B}_{n} \mathcal{C}_{n}} \frac{x^{n k}}{\left(q^{k} ; q^{k}\right)_{n-1}}
$$

Next operating by $\Phi_{h, g}^{(\alpha, \beta, \sigma ; 1)}$, one gets

$$
\begin{aligned}
\Phi_{h, g}^{(\alpha, \beta, \sigma ; 1)} \Theta \mathcal{Y}= & \sum_{n=1}^{[n / \delta]} \frac{\mathcal{H}_{n} \mathcal{G}_{n}}{\mathcal{B}_{n}\left(q^{k} ; q^{k}\right)_{n-1}} \frac{\left\{\prod_{h=0}^{\alpha-1} \prod_{g=0}^{\alpha-1}\left(\Theta+\sigma^{-h} q^{1-(\beta+g) / \alpha}-1\right)\right\}}{\left\{\prod_{h=0}^{\alpha-1} \prod_{g=0}^{\alpha-1}\left(\sigma^{-h} q^{1-(\beta+g) / \alpha}\right)\right\}} \\
& \times\left\{\prod_{h=0}^{\alpha-1} \prod_{g=0}^{\alpha-1}\left(\sigma^{h} q^{(\beta+g) / \alpha} ; q\right)_{n}\right\}^{-1} x^{n k} \\
= & \sum_{n=1}^{[n / \delta]} \frac{\mathcal{H}_{n} \mathcal{G}_{n}}{\mathcal{B}_{n}\left(q^{k} ; q^{k}\right)_{n-1}} \frac{\left\{\prod_{h=0}^{\alpha-1} \prod_{g=0}^{\alpha-1}\left(1-\sigma^{h} q^{n-1+(\beta+g) / \alpha}\right)\right\}}{\left\{\prod_{h=0}^{\alpha-1} \prod_{g=0}^{\alpha-1}\left(\sigma^{h} q^{(\beta+g) / \alpha} ; q\right)_{n}\right\}} x^{n k} \\
= & \sum_{n=1}^{[n / \delta]} \frac{\mathcal{H}_{n} \mathcal{G}_{n}}{\mathcal{B}_{n} \mathcal{C}_{n-1}\left(q^{k} ; q^{k}\right)_{n-1}} x^{n k} .
\end{aligned}
$$

Finally,

$$
\begin{aligned}
& \Phi_{\ell, \kappa}^{(\mu, \lambda, \eta ; r)} \Phi_{h, g}^{(\alpha, \beta, \sigma ; 1)} \Theta \mathcal{Y} \\
= & \sum_{n=1}^{[n / \delta]} \frac{\mathcal{H}_{n} \mathcal{G}_{n}}{\mathcal{C}_{n-1}\left(q^{k} ; q^{k}\right)_{n-1}} \frac{\left\{\prod_{\ell=0}^{\mu-1} \prod_{\kappa=0}^{\mu-1}\left[\left(\Theta+\eta^{-\ell} q^{1-(\lambda+\kappa) / \mu}-1\right)\right]^{r}\right\}}{\left\{\prod_{\ell=0}^{\mu-1} \prod_{\kappa=0}^{\mu-1}\left[\left(\eta^{-\ell} q^{1-(\lambda+\kappa) / \mu}\right)\right]^{r}\right\}} \\
& \times\left\{\prod_{\ell=0}^{\mu-1} \prod_{\kappa=0}^{\mu-1}\left[\left(\eta^{\ell} q^{(\lambda+\kappa) / \mu} ; q\right)_{n}\right]^{r}\right\}^{-1} x^{n k}
\end{aligned}
$$




$$
\begin{aligned}
= & \sum_{n=1}^{[n / \delta]} \frac{\mathcal{H}_{n} \mathcal{G}_{n}}{\mathcal{C}_{n-1}\left(q^{k} ; q^{k}\right)_{n-1}} \frac{\left\{\prod_{\ell=0}^{\mu-1} \prod_{\kappa=0}^{\mu-1}\left[\left(-q^{n}+\eta^{-\ell} q^{1-(\lambda+\kappa) / \mu}\right)\right]^{r}\right\}}{\left\{\prod_{\ell=0}^{\mu-1} \prod_{\kappa=0}^{\mu-1}\left[\left(\eta^{-\ell} q^{1-(\lambda+\kappa) / \mu}\right)\right]^{r}\right\}} \\
& \times\left\{\prod_{\ell=0}^{\mu-1} \prod_{\kappa=0}^{\mu-1}\left[\left(\eta^{\ell} q^{(\lambda+\kappa) / \mu} ; q\right)_{n}\right]^{r}\right\}^{-1} x^{n k} \\
= & \sum_{n=1}^{[n / \delta]} \frac{\mathcal{H}_{n} \mathcal{G}_{n}}{\mathcal{B}_{n-1} \mathcal{C}_{n-1}\left(q^{k} ; q^{k}\right)_{n-1}} x^{n k} .
\end{aligned}
$$

Thus,

$$
\Phi_{\ell, \kappa}^{(\mu, \lambda, \eta ; r)} \Phi_{h, g}^{(\alpha, \beta, \sigma ; 1)} \Theta \mathcal{Y}=\sum_{n=0}^{[n / \delta]} \frac{\mathcal{H}_{n+1} \mathcal{G}_{n+1}}{\mathcal{B}_{n} \mathcal{C}_{n}\left(q^{k} ; q^{k}\right)_{n}} x^{n k+k}
$$

Further,

$$
\begin{aligned}
& \Psi_{j, i}^{(\delta k,-m k, \chi ; s)} B_{m^{*}}^{(\alpha, \beta, \lambda, \mu)}\left(x^{k} q^{s(k \delta)^{2}} ; s, r \mid q\right) \\
= & \sum_{n=0}^{[n / \delta]} \frac{\mathcal{H}_{n} \mathcal{G}_{n} q^{s(k \delta)^{2} n}}{\mathcal{B}_{n} \mathcal{C}_{n}\left(q^{k} ; q^{k}\right)_{n}} \frac{\left\{\prod_{j=0}^{\delta k-1} \prod_{i=0}^{\delta k-1}\left[\left(\Theta+\chi^{-j} q^{-(-m k+i) /(\delta k)}-1\right)\right]^{s}\right\}}{\left\{\prod_{j=0}^{\delta k-1} \prod_{i=0}^{\delta k-1}\left[\left(\chi^{-j} q^{-(-m k+i) /(\delta k)}\right)\right]^{s}\right\}} x^{n k} \\
= & \sum_{n=0}^{[n / \delta]} \frac{\mathcal{G}_{n} q^{s(k \delta)^{2}}}{\mathcal{B}_{n} \mathcal{C}_{n}\left(q^{k} ; q^{k}\right)_{n}}\left\{\prod_{j=0}^{\delta k-1} \prod_{i=0}^{\delta k-1}\left[\left(\chi^{j} q^{(-m k+i) /(\delta k)} ; q\right)_{n}\right]^{s}\right\} \\
& \times\left\{\prod_{j=0}^{\delta k-1} \prod_{i=0}^{\delta k-1}\left[\left(1-\chi^{j} q^{n+(-m k+i) /(\delta k)}\right)\right]^{s}\right\} x^{n k},
\end{aligned}
$$

and hence

$$
\begin{aligned}
& x^{k} q^{s(k \delta(k \delta-1) / 2)+s k \delta m} \Psi_{j, i}^{(\delta k,-m k, \chi ; s)} B_{m^{*}}^{(\alpha, \beta, \lambda, \mu)}\left(x^{k} q^{s(k \delta)^{2}} ; s, r \mid q\right) \\
= & \sum_{n=0}^{[n / \delta]} \frac{\mathcal{H}_{n+1} \mathcal{G}_{n+1}}{\mathcal{B}_{n} \mathcal{C}_{n}\left(q^{k} ; q^{k}\right)_{n}} x^{n k+k} .
\end{aligned}
$$

The equation (3.1) now follows by comparing (3.3) and (3.4).

\section{Generating function inequality}

With the motivation of work done by ([36], [9], [7], [8], [1], [2], [3]) on inequalities, in this section certain inequalities containing $q$-GKP are obtained. 
Theorem 4.1. If $\alpha, \beta, \lambda>0, m, \delta, \mu, k, s \in \mathbf{N}, r \in \mathbf{N} \cup\{0\}, 0<s t<1$ then the following series inequality holds.

$$
\begin{aligned}
\sum_{m=0}^{\infty} \frac{B_{m^{*}}^{(\alpha, \beta, \lambda, \mu)}\left(x^{k} ; s, r \mid q\right)}{\left(q^{\beta+1} ; q\right)_{\alpha m}} t^{m s} \leq & \left(e_{q^{k}}(t)\right)^{s} \frac{\left[\left(q^{k} ; q^{k}\right)_{\infty}\right]^{s}}{\left(q^{\beta+1} ; q\right)_{\infty}} \\
& \times B_{\infty}^{(\alpha, \beta, \lambda, \mu)}\left(x^{k} t^{s \delta} ; s, r \mid q\right) .
\end{aligned}
$$

Proof. From left hand side of (4.1),

$$
\begin{aligned}
& \sum_{m=0}^{\infty} \frac{B_{m^{*}}^{(\alpha, \beta, \lambda, \mu)}\left(x^{k} ; s, r \mid q\right)}{\left(q^{\beta+1} ; q\right)_{\alpha m}} t^{m s} \\
= & \sum_{m=0}^{\infty} \frac{1}{\left(q^{\beta+1} ; q\right)_{\alpha m}} \frac{\left(q^{\beta+1} ; q\right)_{\alpha m}}{\left[\left(q^{k} ; q^{k}\right)_{m}\right]^{s}} \\
& \times \sum_{n=0}^{m^{*}} \frac{q^{s k \delta n(m+(\delta n k-1) / 2)} q^{\delta n(\alpha(\beta+1)+r \mu \lambda)}\left[\left(q^{-m k} ; q^{k}\right)_{\delta n}\right]^{s} x^{k n}}{\left(q^{\beta+1} ; q\right)_{\alpha n}\left[\left(q^{\lambda} ; q\right)_{\mu n}\right]^{r}\left(q^{k} ; q^{k}\right)_{n}} t^{m s} \\
= & \sum_{m=0}^{\infty} \sum_{n=0}^{m^{*}} \frac{q^{s k \delta n(m+(\delta n k-1) / 2)} q^{\delta n(\alpha(\beta+1)+r \mu \lambda)} x^{k n}}{\left[\left(q^{k} ; q^{k}\right)_{m}\right]^{s}\left(q^{\beta+1} ; q\right)_{\alpha n}\left[\left(q^{\lambda} ; q\right)_{\mu n}\right]^{r}\left(q^{k} ; q^{k}\right)_{n}} \\
& \times \frac{(-1)^{s \delta n} q^{s k \delta n(\delta n-1) / 2-s k m \delta n}\left[\left(q^{k} ; q^{k}\right)_{m}\right]^{s}}{\left[\left(q^{k} ; q^{k}\right)_{(m-\delta n)}\right]^{s}} \\
= & \sum_{m=0}^{\infty} \sum_{n=0}^{m^{*}} \frac{(-1)^{s \delta n} q^{s k \delta n(k \delta n-1) / 2+s k \delta n(\delta n-1) / 2} q^{\delta n(\alpha(\beta+1)+r \mu \lambda)} x^{k n}}{\left(q^{\beta+1} ; q\right)_{\alpha n}\left[\left(q^{\lambda} ; q\right)_{\mu n}\right]^{r}\left(q^{k} ; q^{k}\right)_{n}} \\
& \times \frac{t^{m s}}{\left[\left(q^{k} ; q^{k}\right)_{(m-\delta n)}\right]^{s}} \\
& \sum_{m=0}^{\infty} \frac{t^{m s}}{\left[\left(q^{k} ; q^{k}\right)_{m}\right]^{s}} \sum_{n=0}^{\infty} \frac{(-1)^{s \delta n} q^{s k \delta n(k \delta n-1) / 2+s k \delta n(\delta n-1) / 2} q^{\delta n(\alpha(\beta+1)+r \mu \lambda)}}{\left(q^{\beta+1} ; q\right)_{\alpha n}\left[\left(q^{\lambda} ; q\right)_{\mu n}\right]^{r}} \\
& \times t^{s \delta n} x^{k n} \\
& i n q_{n}^{k}
\end{aligned}
$$

Here the inner sum is obtained by making limit $m \rightarrow \infty$ in

$$
\begin{aligned}
& B_{m^{*}}^{(\alpha, \beta, \lambda, \mu)}\left(x^{k} t^{s \delta} ; s, r \mid q\right) \\
= & \frac{\left(q^{\beta+1} ; q\right)_{\alpha m}}{\left[\left(q^{k} ; q^{k}\right)_{m}\right]^{s}} \sum_{n=0}^{m^{*}} \frac{t^{s \delta n} q^{s k \delta n(m+(\delta n k-1) / 2)} q^{\delta n(\alpha(\beta+1)+r \mu \lambda)}\left[\left(q^{-m k} ; q^{k}\right)_{\delta n}\right]^{s} x^{k n}}{\left(q^{\beta+1} ; q\right)_{\alpha n}\left[\left(q^{\lambda} ; q\right)_{\mu n}\right]^{r}\left(q^{k} ; q^{k}\right)_{n}},
\end{aligned}
$$

and since $0<t<1$,

$$
\begin{gathered}
\sum_{m=0}^{\infty} \frac{B_{m^{*}}^{(\alpha, \beta, \lambda, \mu)}\left(x^{k} ; s, r \mid q\right)}{\left(q^{\beta+1} ; q\right)_{\alpha m}} t^{m s}=\sum_{m=0}^{\infty} \frac{t^{m s}}{\left[\left(q^{k} ; q^{k}\right)_{m}\right]^{s}} \frac{\left[\left(q^{k} ; q^{k}\right)_{\infty}\right]^{s}}{\left(q^{\beta+1} ; q\right)_{\infty}} B_{\infty}^{(\alpha, \beta, \lambda, \mu)}\left(x^{k} t^{s \delta} ; s, r \mid q\right) \\
\leq\left(\sum_{m=0}^{\infty} \frac{t^{m}}{\left(q^{k} ; q^{k}\right)_{m}}\right)^{s} \frac{\left[\left(q^{k} ; q^{k}\right)_{\infty}\right]^{s}}{\left(q^{\beta+1} ; q\right)_{\infty}} B_{\infty}^{(\alpha, \beta, \lambda, \mu)}\left(x^{k} t^{s \delta} ; s, r \mid q\right)
\end{gathered}
$$




$$
=\left(e_{q^{k}}(t)\right)^{s} \frac{\left[\left(q^{k} ; q^{k}\right)_{\infty}\right]^{s}}{\left(q^{\beta+1} ; q\right)_{\infty}} B_{\infty}^{(\alpha, \beta, \lambda, \mu)}\left(x^{k} t^{s \delta} ; s, r \mid q\right) .
$$

\subsection{Special cases - Generating function relations}

For $s=1$, the series inequality relations in Theorem 4.1 will yield the generating function relation. Their various specializations are deduced here.

(i) Taking $\alpha=k \in \mathbf{N}, r=0$ in (4.1) leads to

$$
\sum_{m=0}^{\infty} \frac{L_{m^{*}}^{(k, \beta)}\left(x^{k} \mid q\right)}{\left(q^{\beta+1} ; q\right)_{k m}} t^{m}=e_{q^{k}}(t) \frac{\left(q^{k} ; q^{k}\right)_{\infty}}{\left(q^{\beta+1} ; q\right)_{\infty}} L_{\infty}^{(k, \beta)}\left(x^{k} t^{\delta} \mid q\right) .
$$

Further the case $\delta=1$, gives

$$
\sum_{m=0}^{\infty} \frac{Z_{m}^{\beta}(x ; k \mid q)}{\left(q^{\beta+1} ; q\right)_{k m}} t^{m}=e_{q^{k}}(t) \frac{\left(q^{k} ; q^{k}\right)_{\infty}}{\left(q^{\beta+1} ; q\right)_{\infty}} Z_{\infty}^{\beta}\left(x t^{\frac{1}{k}} ; k \mid q\right) .
$$

Finally, for $k=1$ this reduces to (cf. [30, Eq. (1), p. 201])

$$
\sum_{m=0}^{\infty} \frac{L_{m}^{(\beta)}(x \mid q)}{\left(q^{\beta+1} ; q\right)_{m}} t^{m}=e_{q}(t) \frac{(q ; q)_{\infty}}{\left(q^{\beta+1} ; q\right)_{\infty}} L_{\infty}^{(\beta)}(x t \mid q) .
$$

\subsection{Special cases-Inequalities}

If $\alpha=k \in \mathbf{N}$ in (4.1) then

$$
\sum_{m=0}^{\infty} \frac{B_{m^{*}}^{(k, \beta, \lambda, \mu)}\left(x^{k} ; s, r \mid q\right)}{\left(q^{\beta+1} ; q\right)_{k m}} t^{m s} \leq\left(e_{q^{k}}(t)\right)^{s} \frac{\left[\left(q^{k} ; q^{k}\right)_{\infty}\right]^{s}}{\left(q^{\beta+1} ; q\right)_{\infty}} B_{\infty}^{(k, \beta, \lambda, \mu)}\left(x^{k} t^{s \delta} ; s, r \mid q\right) .
$$

This will be used in the next section.

Further for $\delta=1, r=0$, this reduces to

$$
\sum_{m=0}^{\infty} \frac{Z_{m, s}^{\beta}\left(x^{k} \mid q\right)}{\left(q^{\beta+1} ; q\right)_{k m}} t^{m s} \leq\left(e_{q^{k}}(t)\right)^{s} \frac{\left[\left(q^{k} ; q^{k}\right)_{\infty}\right]^{s}}{\left(q^{\beta+1} ; q\right)_{\infty}} Z_{\infty}^{\beta}\left(x^{k} t^{s} ; \mid q\right) .
$$

Consequently, the generalized Laguerre polynomial case $k=1$, is

$$
\sum_{m=0}^{\infty} \frac{L_{m, s}^{(\beta)}(x \mid q)}{\left(q^{\beta+1} ; q\right)_{m}} t^{m s} \leq\left(e_{q}(t)\right)^{s} \frac{\left[(q ; q)_{\infty}\right]^{s}}{\left(q^{\beta+1} ; q\right)_{\infty}} L_{\infty, s}^{(\beta)}\left(x t^{s} \mid q\right)
$$

Here

$$
\begin{aligned}
Z_{m, s}^{\beta}\left(x^{k} \mid q\right)= & \frac{\left(q^{\beta+1} ; q\right)_{k m}}{\left[\left(q^{k} ; q^{k}\right)_{m}\right]^{s}} \sum_{n=0}^{m} \frac{q^{s(k n(k n-1) / 2+k n m)} q^{n(k(\beta+1))}}{\left(q^{\beta+1} ; q\right)_{k n}} \\
& \times \frac{\left[\left(q^{-m k} ; q^{k}\right)_{n}\right]^{s} x^{k n}}{\left(q^{k} ; q^{k}\right)_{n}}, \Re(\beta)>-1,
\end{aligned}
$$

is $q$-extended Konhauser polynomial. And

$$
L_{m, s}^{(\beta)}(x \mid q)=Z_{m, s}^{\beta}(x \mid q) .
$$

is $q$-extended Laguerre polynomial. 


\section{Finite $q$-series inequality}

In this section, the inequality below involves finite $q$-series and $q$-GKP.

Theorem 5.1. If $\beta, \lambda>0, m, \delta, \mu, k, s \in \mathbf{N}, r \in \mathbf{N} \cup\{0\}$, then

$$
\begin{aligned}
B_{m^{*}}^{(k, \beta, \lambda, \mu)}\left(x^{k} ; s, r \mid q\right) & \leq\left(q^{\beta+1} ; q\right)_{k m}\left(\frac{x}{y}\right)^{\frac{k m}{\delta}} \sum_{j=0}^{m} \frac{(-1)^{j} q^{k j(j-1) / 2}}{\left(q^{k} ; q^{k}\right)_{j}} \\
& \times\left(\left(\frac{y}{x}\right)^{\frac{k}{\delta}} q^{k(-j-s+1)} ; q^{k}\right)_{j} \frac{B_{(m-j)^{*}}^{(k, \beta, \lambda, \mu)}\left(y^{k} ; s, r \mid q\right)}{\left(q^{\beta+1} ; q\right)_{k(m-j)}} .
\end{aligned}
$$

Proof. From the inequality (4.2), one gets

$$
\sum_{m=0}^{\infty} \frac{B_{m^{*}}^{(k, \lambda, \mu)}\left(x^{k} ; s, r \mid q\right)}{\left(q^{\beta+1} ; q\right)_{k m}} t^{m s} \leq\left(e_{q^{k}}(t)\right)^{s} \frac{\left[\left(q^{k} ; q^{k}\right)_{\infty}\right]^{s}}{\left(q^{\beta+1} ; q\right)_{\infty}} B_{\infty}^{(k, \beta, \lambda, \mu)}\left(x^{k} t^{s \delta} ; s, r \mid q\right) .
$$

With $t=\left(\frac{y}{k}\right)^{\frac{k}{s \delta}} w$, it gives

$$
\begin{aligned}
& \sum_{m=0}^{\infty} \frac{B_{m^{*}}^{(k, \beta, \lambda, \mu)}\left(x^{k} ; s, r \mid q\right)}{\left(q^{\beta+1} ; q\right)_{k m}}\left(\frac{y}{k}\right)^{\frac{k m}{\delta}} w^{m s} \\
\leq & \left(e_{q^{k}}\left(\left(\frac{y}{k}\right)^{\frac{k}{s \delta}} w\right)\right)^{s} \frac{\left[\left(q^{k} ; q^{k}\right)_{\infty}\right]^{s}}{\left(q^{\beta+1} ; q\right)_{\infty}} B_{\infty}^{(k, \beta, \lambda, \mu)}\left(\left(\frac{x y}{k}\right)^{k} w^{s \delta} ; s, r \mid q\right) .
\end{aligned}
$$

Hence,

$$
\begin{aligned}
& \sum_{m=0}^{\infty} \frac{B_{m^{*}}^{(k, \beta, \lambda, \mu)}\left(x^{k} ; s, r \mid q\right)}{\left(q^{\beta+1} ; q\right)_{k m}}\left(\frac{y}{k}\right)^{\frac{k m}{\delta}} w^{m s}\left(e_{q^{k}}\left(\left(\frac{y}{k}\right)^{\frac{k}{s \delta}} w\right)\right)^{-s} \\
\leq & \frac{\left[\left(q^{k} ; q^{k}\right)_{\infty}\right]^{s}}{\left(q^{\beta+1} ; q\right)_{\infty}} B_{\infty}^{(k, \beta, \lambda, \mu)}\left(\left(\frac{x y}{k}\right)^{k} w^{s \delta} ; s, r \mid q\right) .
\end{aligned}
$$

Now interchanging the role of $x$ and $y$ in (5.2), it yields

$$
\begin{aligned}
& \sum_{m=0}^{\infty} \frac{B_{m^{*}}^{(k, \beta, \lambda, \mu)}\left(y^{k} ; s, r \mid q\right)}{\left(q^{\beta+1} ; q\right)_{k m}}\left(\frac{x}{k}\right)^{\frac{k m}{\delta}} w^{m s}\left(e_{q^{k}}\left(\left(\frac{x}{k}\right)^{\frac{k}{s \delta}} w\right)\right)^{-s} \\
\leq & \frac{\left[\left(q^{k} ; q^{k}\right)_{\infty}\right]^{s}}{\left(q^{\beta+1} ; q\right)_{\infty}} B_{\infty}^{(k, \beta, \lambda, \mu)}\left(\left(\frac{x y}{k}\right)^{k} w^{s \delta} ; s, r \mid q\right) .
\end{aligned}
$$

Here from (5.2) and (5.3), either

$$
\begin{aligned}
& \sum_{m=0}^{\infty} \frac{B_{m^{*}}^{(k, \beta, \lambda, \mu)}\left(x^{k} ; s, r \mid q\right)}{\left(q^{\beta+1} ; q\right)_{k m}}\left(\frac{y}{k}\right)^{\frac{k m}{\delta}} w^{m s}\left(e_{q^{k}}\left(\left(\frac{y}{k}\right)^{\frac{k}{s \delta}} w\right)\right)^{-s} \\
\leq & \sum_{m=0}^{\infty} \frac{B_{m^{*}}^{(k, \beta, \lambda, \mu)}\left(y^{k} ; s, r \mid q\right)}{\left(q^{\beta+1} ; q\right)_{k m}}\left(\frac{x}{k}\right)^{\frac{k m}{\delta}} w^{m s}\left(e_{q^{k}}\left(\left(\frac{x}{k}\right)^{\frac{k}{s \delta}} w\right)\right)^{-s}
\end{aligned}
$$


or

$$
\begin{aligned}
& \sum_{m=0}^{\infty} \frac{B_{m^{*}}^{(k, \lambda, \mu)}\left(y^{k} ; s, r \mid q\right)}{\left(q^{\beta+1} ; q\right)_{k m}}\left(\frac{x}{k}\right)^{\frac{k m}{\delta}} w^{m s}\left(e_{q^{k}}\left(\left(\frac{x}{k}\right)^{\frac{k}{s \delta}} w\right)\right)^{-s} \\
\leq & \sum_{m=0}^{\infty} \frac{B_{m^{*}}^{(k, \lambda, \mu)}\left(x^{k} ; s, r \mid q\right)}{\left(q^{\beta+1} ; q\right)_{k m}}\left(\frac{y}{k}\right)^{\frac{k m}{\delta}} w^{m s}\left(e_{q^{k}}\left(\left(\frac{y}{k}\right)^{\frac{k}{s \delta}} w\right)\right)^{-s}
\end{aligned}
$$

Now rewriting the inequality (5.4) in the form

$$
\begin{aligned}
& \sum_{m=0}^{\infty} \frac{B_{m^{*}}^{(k, \beta, \lambda, \mu)}\left(x^{k} ; s, r \mid q\right)}{\left(q^{\beta+1} ; q\right)_{k m}}\left(\frac{y}{k}\right)^{\frac{k m}{\delta}} w^{m s} \\
\leq & \sum_{m=0}^{\infty} \frac{B_{m^{*}}^{(k, \beta, \lambda, \mu)}\left(y^{k} ; s, r \mid q\right)}{\left(q^{\beta+1} ; q\right)_{k m}}\left(\frac{x}{k}\right)^{\frac{k m}{\delta}} w^{m s}\left(e_{q^{k}}\left(\left(\frac{y}{k}\right)^{\frac{k}{s \delta}} w\right)\right)^{s} \\
& \times\left(e_{q^{k}}\left(\left(\frac{x}{k}\right)^{\frac{k}{s \delta}} w\right)\right)^{-s}
\end{aligned}
$$

and using the easily verifiable identities and inequalities $(s x, s y \in(0,1), s \in \mathbf{N}),([11]$, $[13])$ :

$$
\begin{aligned}
e_{q}(x) E_{q}(-x) & =1 \\
\left(E_{q}(-x)\right)^{s} & \leq E_{q}\left(-x^{s}\right) \\
(1+x) E_{q}(q x) & =E_{q}(x) \\
e_{q^{-1}}(x) & =E_{q}(-x q) \\
(1-x) e_{q}(x) & =e_{q}(q x) \\
\left(e_{q^{-1}}\left(-x q^{-1}\right)\right)^{-s} & \leq e_{q}\left(x^{s} q^{-s}\right), \\
\left(e_{q}(-x)\right)^{s} & \leq e_{q}\left(-x^{s}\right) \\
\left(e_{q^{-1}}\left(-x q^{-1}\right)\right)^{s} & \leq e_{q}\left(-x^{s} q^{-s}\right),
\end{aligned}
$$

the above inequality can easily be written as

$$
\begin{aligned}
& \sum_{m=0}^{\infty} \frac{B_{m^{*}}^{(k, \beta, \lambda, \mu)}\left(x^{k} ; s, r \mid q\right)}{\left(q^{\beta+1} ; q\right)_{k m}}\left(\frac{y}{k}\right)^{\frac{k m}{\delta}} w^{m s} \\
\leq & \sum_{m=0}^{\infty} \frac{B_{m^{*}}^{(k, \beta, \lambda, \mu)}\left(y^{k} ; s, r \mid q\right)}{\left(q^{\beta+1} ; q\right)_{k m}}\left(\frac{x}{k}\right)^{\frac{k m}{\delta}} w^{m s}\left(E_{q^{k}}\left(-\left(\frac{x}{k}\right)^{\frac{k}{s \delta}} w\right)\right)^{s} \\
& \times\left(E_{q^{k}}\left(-\left(\frac{y}{k}\right)^{\frac{k}{s \delta}} w\right)\right)^{-s} \\
\leq & \sum_{m=0}^{\infty} \frac{B_{m^{*}}^{(k, \beta, \lambda, \mu)}\left(y^{k} ; s, r \mid q\right)}{\left(q^{\beta+1} ; q\right)_{k m}}\left(\frac{x}{k}\right)^{\frac{k m}{\delta}} w^{m s} E_{q^{k}}\left(-\left(\frac{x}{k}\right)^{\frac{k}{\delta}} w^{s}\right) \\
& \times\left(E_{q^{k}}\left(-\left(\frac{y}{k}\right)^{\frac{k}{s \delta}} w\right)\right)^{-s}
\end{aligned}
$$




$$
\begin{aligned}
& =\sum_{m=0}^{\infty} \frac{B_{m^{*}}^{(k, \lambda, \mu)}\left(y^{k} ; s, r \mid q\right)}{\left(q^{\beta+1} ; q\right)_{k m}}\left(\frac{x}{k}\right)^{\frac{k m}{\delta}} w^{m s} E_{q^{k}}\left(-\left(\frac{x}{k}\right)^{\frac{k}{\delta}} w^{s}\right) \\
& \times\left(1-\left(\frac{y}{k}\right)^{\frac{k}{s \delta}} w\right)^{-s}\left(E_{q^{k}}\left(-q^{k}\left(\frac{y}{k}\right)^{\frac{k}{s \delta}} w\right)\right)^{-s} \\
& =\sum_{m=0}^{\infty} \frac{B_{m^{*}}^{(k, \beta, \mu)}\left(y^{k} ; s, r \mid q\right)}{\left(q^{\beta+1} ; q\right)_{k m}}\left(\frac{x}{k}\right)^{\frac{k m}{\delta}} w^{m s} E_{q^{k}}\left(-\left(\frac{x}{k}\right)^{\frac{k}{\delta}} w^{s}\right) \\
& \times\left(1-\left(\frac{y}{k}\right)^{\frac{k}{s \delta}} w\right)^{-s}\left(e_{q^{-k}}\left(\left(\frac{y}{k}\right)^{\frac{k}{s \delta}} w\right)\right)^{-s} \\
& =\sum_{m=0}^{\infty} \frac{B_{m^{*}}^{(k, \beta, \lambda)}\left(y^{k} ; s, r \mid q\right)}{\left(q^{\beta+1} ; q\right)_{k m}}\left(\frac{x}{k}\right)^{\frac{k m}{\delta}} w^{m s} E_{q^{k}}\left(-\left(\frac{x}{k}\right)^{\frac{k}{\delta}} w^{s}\right) \\
& \times\left(e_{q^{-k}}\left(q^{-k}\left(\frac{y}{k}\right)^{\frac{k}{s \delta}} w\right)\right)^{-s} \\
& \leq \sum_{m=0}^{\infty} \frac{B_{m^{*}}^{(k, \beta, \lambda, \mu)}\left(y^{k} ; s, r \mid q\right)}{\left(q^{\beta+1} ; q\right)_{k m}}\left(\frac{x}{k}\right)^{\frac{k m}{\delta}} w^{m s} E_{q^{k}}\left(-\left(\frac{x}{k}\right)^{\frac{k}{\delta}} w^{s}\right) \\
& \times e_{q^{k}}\left(q^{-s k}\left(\frac{y}{k}\right)^{\frac{k}{\delta}} w^{s}\right) \\
& =\sum_{m=0}^{\infty} \frac{B_{m^{*}}^{(k, \beta, \lambda, \mu)}\left(y^{k} ; s, r \mid q\right)}{\left(q^{\beta+1} ; q\right)_{k m}}\left(\frac{x}{k}\right)^{\frac{k m}{\delta}} w^{m s} \sum_{j=0}^{\infty} \frac{(-1)^{j} q^{k j(j-1) / 2}}{\left(q^{k} ; q^{k}\right)_{j}}\left(\frac{x}{k}\right)^{\frac{k j}{\delta}} w^{s j} \\
& \times \sum_{i=0}^{\infty} \frac{\left(\frac{y}{k}\right)^{\frac{k i}{\delta}} q^{-s k i} w^{s i}}{\left(q^{k} ; q^{k}\right)_{i}} \\
& =\sum_{m=0}^{\infty} \frac{B_{m^{*}}^{(k, \beta, \lambda, \mu)}\left(y^{k} ; s, r \mid q\right)}{\left(q^{\beta+1} ; q\right)_{k m}}\left(\frac{x}{k}\right)^{\frac{k m}{\delta}} w^{m s} \sum_{j=0}^{\infty} \sum_{i=0}^{j} \frac{(-1)^{j-i} q^{k(j-i)(j-i-1) / 2}}{\left(q^{k} ; q^{k}\right)_{j-i}} \\
& \times\left(\frac{x}{k}\right)^{\frac{k(j-i)}{\delta}} \frac{w^{s(j-i)}\left(\frac{y}{k}\right)^{\frac{k i}{\delta}} q^{-s k i} w^{s i}}{\left(q^{k} ; q^{k}\right)_{i}} \\
& =\sum_{m=0}^{\infty} \frac{B_{m^{*}}^{(k, \beta, \lambda)}\left(y^{k} ; s, r \mid q\right)}{\left(q^{\beta+1} ; q\right)_{k m}}\left(\frac{x}{k}\right)^{\frac{k m}{\delta}} w^{m s} \sum_{j=0}^{\infty} \frac{(-1)^{j} q^{k j(j-1) / 2} w^{s j}}{\left(q^{k} ; q^{k}\right)_{j}}\left(\frac{x}{k}\right)^{\frac{k j}{\delta}} \\
& \times \sum_{i=0}^{j}(-1)^{i} q^{k i(i-1) / 2}\left[\begin{array}{l}
j \\
i
\end{array}\right]_{k}\left(\frac{y}{x}\right)^{\frac{k i}{\delta}} q^{(1-j-s) k i} \\
& =\sum_{m=0}^{\infty} \frac{B_{m^{*}}^{(k, \beta, \lambda, \mu)}\left(y^{k} ; s, r \mid q\right)}{\left(q^{\beta+1} ; q\right)_{k m}}\left(\frac{x}{k}\right)^{\frac{k m}{\delta}} w^{m s} \sum_{j=0}^{\infty} \frac{(-1)^{j} q^{k j(j-1) / 2} w^{s j}}{\left(q^{k} ; q^{k}\right)_{j}}\left(\frac{x}{k}\right)^{\frac{k j}{\delta}} \\
& \times \prod_{i=1}^{j}\left(1-\left(\frac{y}{x}\right)^{\frac{k}{\delta}} q^{k(i-j-s)}\right)
\end{aligned}
$$




$$
\begin{aligned}
= & \sum_{m=0}^{\infty} \sum_{j=0}^{m} \frac{B_{(m-j)^{*}}^{(k, \beta, \lambda, \mu)}\left(y^{k} ; s, r \mid q\right)}{\left(q^{\beta+1} ; q\right)_{k(m-j)}}\left(\frac{x}{k}\right)^{\frac{k(m-j)}{\delta}} w^{(m-j) s} \frac{q^{k j(j-1) / 2} w^{s j}}{\left(q^{k} ; q^{k}\right)_{j}}\left(\frac{x}{k}\right)^{\frac{k j}{\delta}} \\
& \times \prod_{i=1}^{j}\left(1-\left(\frac{y}{x}\right)^{\frac{k}{\delta}} q^{k(i-j)}\right) \\
= & \sum_{m=0}^{\infty} \sum_{j=0}^{m} \frac{B_{(m-j)^{*}}^{(k, \beta, \lambda, \mu)}\left(y^{k} ; s, r \mid q\right)}{\left(q^{\beta+1} ; q\right)_{k(m-j)}} \frac{q^{k j(j-1) / 2}}{\left(q^{k} ; q^{k}\right)_{j}}\left(\frac{x}{k}\right)^{\frac{k m}{\delta}}\left(\left(\frac{y}{x}\right)^{\frac{k}{\delta}} q^{k(-j-s+1)} ; q^{k}\right)_{j} w^{m s} .
\end{aligned}
$$

Now comparing the coefficients of $w^{m s}$ both the sides, one arrives at (5.1).

\subsection{Special cases}

(i) From (5.1), one gets finite summation formulas for $s=1$ :

$$
\begin{aligned}
B_{m^{*}}^{(k, \beta, \lambda, \mu)}\left(x^{k} ; 1, r \mid q\right)= & \left(q^{\beta+1} ; q\right)_{k m}\left(\frac{x}{y}\right)^{\frac{k m}{\delta}} \sum_{j=0}^{m} \frac{(-1)^{j} q^{k j(j-1) / 2}}{\left(q^{k} ; q^{k}\right)_{j}} \\
& \times\left(\left(\frac{y}{x}\right)^{\frac{k}{\delta}} q^{-k j} ; q^{k}\right)_{j} \frac{B_{(m-j)^{*}}^{(k, \beta, \lambda, \mu)}\left(y^{k} ; 1, r \mid q\right)}{\left(q^{\beta+1} ; q\right)_{k(m-j)}}
\end{aligned}
$$

From (5.4), with $r=0$, the following summation formula involving the generalized Laguerre polynomial (1.31) occurs.

$$
\begin{aligned}
L_{m^{*}}^{(k, \beta)}\left(x^{k} \mid q\right)= & \left(q^{\beta+1} ; q\right)_{k m}\left(\frac{x}{y}\right)^{\frac{k m}{\delta}} \sum_{j=0}^{m} \frac{(-1)^{j} q^{k j(j-1) / 2}}{\left(q^{k} ; q^{k}\right)_{j}}\left(\left(\frac{y}{x}\right)^{\frac{k}{\delta}} q^{-k j} ; q^{k}\right)_{j} \\
& \times \frac{L_{(m-j)^{*}}^{(k, \beta)}\left(y^{k} \mid q\right)}{\left(q^{\beta+1} ; q\right)_{k(m-j)}} .
\end{aligned}
$$

Further, $\delta=1$ in $(5.5)$ provides

$$
\begin{aligned}
Z_{m}^{\beta}(x ; k \mid q)= & \left(q^{\beta+1} ; q\right)_{k m}\left(\frac{x}{y}\right)^{k m} \sum_{j=0}^{m} \frac{(-1)^{j} q^{k j(j-1) / 2}}{\left(q^{k} ; q^{k}\right)_{j}}\left(\left(\frac{y}{x}\right)^{k} q^{-k j} ; q^{k}\right)_{j} \\
& \times \frac{Z_{(m-j)}^{\beta}(y ; k \mid q)}{\left(q^{\beta+1} ; q\right)_{k(m-j)}} .
\end{aligned}
$$

The Laguerre polynomial case follows immediately with $k=1$ in the form:

$$
\begin{aligned}
L_{m}^{(\beta)}(x \mid q)= & \left(q^{\beta+1} ; q\right)_{m}\left(\frac{x}{y}\right)^{m} \sum_{j=0}^{m} \frac{(-1)^{j} q^{j(j-1) / 2}}{(q ; q)_{j}}\left(\left(\frac{y}{x}\right) q^{-j} ; q\right)_{j} \\
& \times \frac{L_{(m-j)}^{(\beta)}(y \mid q)}{\left(q^{\beta+1} ; q\right)_{(m-j)}} .
\end{aligned}
$$




\section{Mixed relation}

Theorem 6.1. For $\beta, \lambda>0, m, \delta, \mu, k, s \in \mathbf{N}, r \in \mathbf{N} \cup\{0\}$ there hold the mixed relations:

$$
\begin{aligned}
& \left(1-q^{\beta}\right) B_{m^{*}}^{(k, \lambda, \lambda, \mu)}\left(x^{k} ; s, r \mid q\right)+(1-q) q^{\beta} x D_{q} B_{m^{*}}^{(k, \beta, \lambda, \mu)}\left(x^{k} ; s, r \mid q\right) \\
= & \left(1-q^{\beta+k m}\right) B_{m^{*}}^{(k, \beta-1, \lambda, \mu)}\left(\left(x q^{\delta}\right)^{k} ; s, r \mid q\right),
\end{aligned}
$$

where

$$
D_{q} f(x)=\frac{f(x)-f(x q)}{x-x q}
$$

Proof. Here

$$
\begin{aligned}
& \text { l.h.s. }=\left(1-q^{\beta}\right) B_{m^{*}}^{(k, \beta, \lambda, \mu)}\left(x^{k} ; s, r \mid q\right)+(1-q) q^{\beta} x D_{q} B_{m^{*}}^{(k, \beta, \lambda, \mu)}\left(x^{k} ; s, r \mid q\right) \\
& =\left(1-q^{\beta}\right) \frac{\left(q^{\beta+1} ; q\right)_{k m}}{\left[\left(q^{k} ; q^{k}\right)_{m}\right]^{s}} \sum_{n=0}^{m^{*}} \frac{q^{s(k \delta n(k \delta n-1) / 2+k \delta n m)} q^{\delta n(k(\beta+1)+r \mu \lambda)}}{\left(q^{\beta+1} ; q\right)_{k n}\left[\left(q^{\lambda} ; q\right)_{\mu n}\right]^{r}} \\
& \times \frac{\left[\left(q^{-m k} ; q^{k}\right)_{\delta n}\right]^{s} x^{k n}}{\left(q^{k} ; q^{k}\right)_{n}}+(1-q) q^{\beta} \times D_{q} \frac{\left(q^{\beta+1} ; q\right)_{\alpha m}}{\left[\left(q^{k} ; q^{k}\right)_{m}\right]^{s}} \\
& \times \sum_{n=0}^{m^{*}} \frac{q^{s(k \delta n(k \delta n-1) / 2+k \delta n m)} q^{\delta n(\alpha(\beta+1)+r \mu \lambda)}}{\left(q^{\beta+1} ; q\right)_{k n}\left[\left(q^{\lambda} ; q\right)_{\mu n}\right]^{r}} \frac{\left[\left(q^{-m k} ; q^{k}\right)_{\delta n}\right]^{s} x^{k n}}{\left(q^{k} ; q^{k}\right)_{n}} \\
& =\left(1-q^{\beta}\right) \frac{\left(q^{\beta+1} ; q\right)_{k m}}{\left[\left(q^{k} ; q^{k}\right)_{m}\right]^{s}} \sum_{n=0}^{m^{*}} \frac{q^{s(k \delta n(k \delta n-1) / 2+k \delta n m)} q^{\delta n(k(\beta+1)+r \mu \lambda)}}{\left(q^{\beta+1} ; q\right)_{k n}\left[\left(q^{\lambda} ; q\right)_{\mu n}\right]^{r}} \\
& \times \frac{\left[\left(q^{-m k} ; q^{k}\right)_{\delta n}\right]^{s} x^{k n}}{\left(q^{k} ; q^{k}\right)_{n}}+(1-q) q^{\beta} x \frac{\left(q^{\beta+1} ; q\right)_{\alpha m}}{\left[\left(q^{k} ; q^{k}\right)_{m}\right]^{s}} \\
& \times \sum_{n=0}^{m^{*}} \frac{q^{s(k \delta n(k \delta n-1) / 2+k \delta n m)} q^{\delta n(\alpha(\beta+1)+r \mu \lambda)}}{\left(q^{\beta+1} ; q\right)_{k n}\left[\left(q^{\lambda} ; q\right)_{\mu n}\right]^{r}} \frac{\left[\left(q^{-m k} ; q^{k}\right)_{\delta n}\right]^{s}}{\left(q^{k} ; q^{k}\right)_{n}} D_{q}\left(x^{k n}\right) \\
& =\left(1-q^{\beta}\right) \frac{\left(q^{\beta+1} ; q\right)_{k m}}{\left[\left(q^{k} ; q^{k}\right)_{m}\right]^{s}} \sum_{n=0}^{m^{*}} \frac{q^{s(k \delta n(k \delta n-1) / 2+k \delta n m)} q^{\delta n(k(\beta+1)+r \mu \lambda)}}{\left(q^{\beta+1} ; q\right)_{k n}\left[\left(q^{\lambda} ; q\right)_{\mu n}\right]^{r}} \\
& \times \frac{\left[\left(q^{-m k} ; q^{k}\right)_{\delta n}\right]^{s} x^{k n}}{\left(q^{k} ; q^{k}\right)_{n}}+(1-q) q^{\beta} x \frac{\left(q^{\beta+1} ; q\right)_{\alpha m}}{\left[\left(q^{k} ; q^{k}\right)_{m}\right]^{s}} \\
& \times \sum_{n=0}^{m^{*}} \frac{q^{s(k \delta n(k \delta n-1) / 2+k \delta n m)} q^{\delta n(\alpha(\beta+1)+r \mu \lambda)}}{\left(q^{\beta+1} ; q\right)_{k n}\left[\left(q^{\lambda} ; q\right)_{\mu n}\right]^{r}} \frac{\left[\left(q^{-m k} ; q^{k}\right)_{\delta n}\right]^{s}}{\left(q^{k} ; q^{k}\right)_{n}} \frac{\left(1-q^{k n}\right)}{x(1-q)}
\end{aligned}
$$




$$
\begin{aligned}
= & \left(1-q^{\beta}\right) \frac{\left(q^{\beta+1} ; q\right)_{k m}}{\left[\left(q^{k} ; q^{k}\right)_{m}\right]^{s}} \sum_{n=0}^{m^{*}} \frac{q^{s(k \delta n(k \delta n-1) / 2+k \delta n m)} q^{\delta n(k(\beta+1)+r \mu \lambda)}}{\left(q^{\beta+1} ; q\right)_{k n}\left[\left(q^{\lambda} ; q\right)_{\mu n}\right]^{r}} \\
& \times \frac{\left[\left(q^{-m k} ; q^{k}\right)_{\delta n}\right]^{s} x^{k n}}{\left(q^{k} ; q^{k}\right)_{n}}+\left(q^{\beta}-q^{k n+\beta}\right) \frac{\left(q^{\beta+1} ; q\right)_{\alpha m}}{\left[\left(q^{k} ; q^{k}\right)_{m}\right]^{s}} \\
& \times \sum_{n=0}^{m^{*}} \frac{q^{s(k \delta n(k \delta n-1) / 2+k \delta n m)} q^{\delta n(\alpha(\beta+1)+r \mu \lambda)}}{\left(q^{\beta+1} ; q\right)_{k n}\left[\left(q^{-m k} ; q^{k}\right)_{\delta n}\right]^{s}} \\
& \frac{\left.\left(1-q^{\beta+k m}\right]^{r}\right)\left(q^{\beta} ; q\right)_{k m}}{\left(q^{k} ; q^{k}\right)_{n}} \sum_{n=0}^{m^{*}} \frac{q^{s(k \delta n(k \delta n-1) / 2+k \delta n m)} q^{\delta n(k(\beta+1)+r \mu \lambda)}}{\left[\left(q^{\beta}\right]_{k n}\left[\left(q^{\lambda} ; q\right)_{\mu n}\right]^{r}\right.} \\
& \times \frac{\left[\left(q^{-m k} ; q^{k}\right)_{\delta n}\right]^{s} x^{k n}}{\left(q^{k} ; q^{k}\right)_{n}} . \\
= & \left(1-q^{\beta+k m}\right) B_{m^{*}}^{(k, \beta-1, \lambda, \mu)}\left(\left(x q^{\delta}\right)^{k} ; s, r \mid q\right) \\
= & \text { r.h.s. }
\end{aligned}
$$

Acknowledgement. The author sincerely thanks her guide Prof. B. I. Dave and the referee(s) for making the valuable suggestions for the improvement of the manuscript.

\section{References}

[1] Agarwal, P., Some inequalities involving Hadamardtype $k$-fractional integral operators, Math. Methods Appl. Sci., 40(2017), no. 11, 3882-3891.

[2] Agarwal, P., Jleli, M., Tomar, M., Certain Hermite-Hadamard type inequalities via generalized $k$-fractional integrals, J. Inequal. Appl., 55(2017), no. 1, 1-10.

[3] Agarwal, P., Tariboon, J., Ntouyas, S.K., Some generalized Riemann-Liouville $k$ fractional integral inequalities, J. Inequal. Appl., 122(2016), no. 1, 1-13.

[4] Agarwal, R.P., Certain fractional q-integrals and q-derivatives, Proc. Comb. Phil. Soc., 66(1969), no. 3, 365-370.

[5] Al-Salam, W.A., Some fractional q-integarls and q-derivatives, Proc. Edinb. Math. Soc., 15(1966), 135-140.

[6] Al-Salam, W.A., Verma, A., q-Konhauser polynomials, Pacific J. Math., 1(1983), no. 108, 1-7.

[7] Baleanu, D., Agarwal, P., Certain inequalities involving the fractional-integral operators, Abstr. Appl. Anal., 2014(2014), 1-10.

[8] Baleanu, D., Purohit, S.D., Agarwal, P., On fractional integral inequalities involving hypergeometric operators, Chinese Journal of Mathematics, 2014(2014), 1-10.

[9] Choi, J.W.A., Agarwal, P., Some new Saigo type fractional integral inequalities and their-analogues, Abstr. Appl. Anal., 2014(2014), 1-11.

[10] Choi, J.W.A., Agarwal, P., A note on fractional integral operator associated with multiindex Mittag-Leffler functions, Filomat, 30(2016), no. 7, 1931-1939.

[11] Clarkson, P.A., Nijhoff, F.W., Symmetries and Integrability of Difference Equations, Cambridge University Press, 1999. 
[12] Dave, B.I., Extensions of Certain Inverse Series Relations and Associated Polynomials, Ph. D. Thesis, The M.S. University of Baroda, Vadodara, 1994.

[13] Gasper, G., Rahman, M., Basic Hypergeometric Series, Cambridge University Press, Cambridge, 1990.

[14] Gorenflo, R., Kilbas, A.A., Rogosin, S.V., On the generalized Mittag-Leffler type function, Integral Transforms Spec. Funct., 7(1998), 215-224.

[15] Gupta, I.S., Debnath, L., A note on fractional integral operator associated with multiindex Mittag-Leffler functions, Integral Transforms Spec. Funct., 18(2007), no. 5, 329-336.

[16] Hahn, W., Über Orthogonal polynome, die q-Differenzengleichungen genügen, Math. Nachr., 2(1949), 4-34.

[17] Haubold, H.J., Mathai, A.M., Saxena, R.K., The H-Function: Theory and Applications, Centre for Mathematical Sciences, Pala Campus, Kerala, India, 2008.

[18] Kilbas, A.A., Saigo, M., Saxena, R.K., Generalized Mittag-Leffler function and generalized fractional calculus operators, Integral Transforms Spec. Funct., 15(2004), 31-49.

[19] Mansour, M., An asymptotic expansion of the q-Gamma function $\Gamma_{q}(x)$, J. Nonlinear Math. Phys., 13(2006), no. 4, 479-483.

[20] Mittag-Leffler, G., Sur la nouvelle fonction $E_{\alpha}(x)$, C.R. Acad. Sci., Paris, 137(1903), 554-558.

[21] Nagai, A., Discrete Mittag-Leffler function and its applications, Deparment of Mathematical Sciences, Graduate School of Engineering Sciences, Osaka University, 1302(2003), 1-20.

[22] Nathwani, B.V., Generalized q-Mittag-Leffler function and its properties, Journal of Divulgaciones Matematicas, 18(2017), no. 1, 10-33.

[23] Nathwani, B.V., Dave, B.I., Generalized Mittag-Leffler function and its properties, Math. Student, 86(2017), no. 1-2, 63-76.

[24] Nathwani, B.V., Dave, B.I., Fractional q-calculus of anextended q-Mittag-Leffler function, J. Fract. Calc. Appl., 9(2018), no. 1, 64-81.

[25] Prabhakar, T.R., Rekha, S., Some results on the polynomials $L_{n}^{\alpha, \beta}(x)$, Rocky Mountain J. Math., 8(1978), no. 4, 751-754.

[26] Prajapati, J.C., Ajudia, N., Agarwal, P., Some results due to Konhauser polynomials of first kind and Laguerre polynomials, Appl. Math. Comput., 247(2014), 639-650.

[27] Prajapati, J.C., Dave, B.I., Nathwani, B.V., On a unification of generalized MittagLeffler function and family of Bessel functions, Advances in Pure Mathematics, 3(2013), 127-137.

[28] Prajapati, J.C., Nathwani, B.V., Recurrence relation of a unified generalized MittagLeffler function, Palest. J. Math., 3(2014), no. 1, 94-98.

[29] Prajapati, J.C., Nathwani, B.V., Fractional calculus of a unified Mittag-Leffler function, Ukrainian Math. J., 66(2015), no. 8, 1267-1280.

[30] Rainville, E.D., Special Functions, Macmillan Co., New York, 1960.

[31] Ruzhansky, M.V., Jecho, Y., Agarwal, P., Area, I., Advances in Real and Complex Analysis with Applications, Springer, Singapore, 2017.

[32] Saigo, M., Kilbas, A.A., On Mittag Leffler type function and applications, Integral Transforms Spec. Funct., 7(1998), 97-112.

[33] Saxena, R.K., Kalla, S., Saxena, R., Multivariate analogue of generatized Mittag-Leffer function, Integral Transforms Spec. Funct., 22(2011), no. 7, 533-548. 
[34] Shukla, A.K., Prajapati, J.C., On a generalization of Mittag-Leffler function and its properties, J. Math. Anal. Appl., 336(2007), no. 2, 797-811.

[35] Tariboon, J., Ntouyas, S.K., Agarwal, P., New concepts of fractional quantum calculus and applications to impulsive fractional q-difference equations, Adv. Difference Equ., 18(2015), 1-19.

[36] Wang, G., Agarwal, P., Chand, M., Certain Grüs type inequalities involving the generalized fractional integral operator, J. Inequal. Appl., 147(2014), no. 1, 1-8.

[37] Wiman, A., Über de fundamental satz in der theoric der funktionen $E_{\alpha}(x)$, Acta Math., 29(1905), 191-201.

[38] Wiman, A., Über die nullstellen der funktionen $E_{\alpha}(x)$, Acta Math., 29(1905), 217-234.

[39] Zhang, X., Agarwal, P., Liu, Z., Peng, H., The general solution for impulsive differential equations with Riemann-Liouville fractional order $q(1,2)$, Open Math., 13(2015), no. 1, $1-23$.

Bharti Vishandas Nathwani

Department of Mathematics,

Amity School of Applied Sciences,

Amity University,

Mumbai-410-206, India

e-mail: bharti.nathwani@yahoo.com 